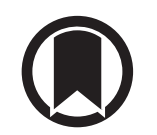

CrossMark

\title{
ERS and ATS diagnostic guidelines for primary ciliary dyskinesia: similarities and differences in approach to diagnosis
}

\author{
Amelia Shoemark $\mathbb{1}^{1,2}$, Sharon Dell ${ }^{3}$, Adam Shapiro ${ }^{4}$ and Jane S. Lucas $\mathbb{1}^{5,6}$ \\ Affiliations: ${ }^{1}$ Dept of Molecular and Cellular Medicine, University of Dundee, Dundee, UK. ${ }^{2}$ Dept of Paediatric \\ Respiratory Medicine, Royal Brompton and Harefield NHS Trust, London, UK. ${ }^{3}$ Division of Respiratory \\ Medicine, Dept of Pediatrics, The Hospital for Sick Children, University of Toronto, Toronto, ON, Canada. \\ ${ }^{4}$ McGill University Health Centre Research Institute, Montreal Children's Hospital, Montreal, QC, Canada. \\ ${ }^{5}$ Primary Ciliary Dyskinesia Centre, NIHR Biomedical Research Centre, University Hospital Southampton NHS \\ Foundation Trust, Southampton, UK. 'University of Southampton Faculty of Medicine, Academic Unit of \\ Clinical and Experimental Medicine, Southampton, UK.
}

Correspondence: Amelia Shoemark, Dept of Molecular and Cellular Medicine, University of Dundee, Dundee, UK. E-mail: a.shoemark@dundee.ac.uk

@ERSpublications

ERS and ATS guidelines for PCD diagnosis present different recommendations. Authors from both guidelines clarify similarities, differences and steps required to develop an internationally agreed pathway. TEM or genotyping confirm a diagnosis of PCD. http://bit.ly/2SR7GWm

Cite this article as: Shoemark A, Dell S, Shapiro A, et al. ERS and ATS diagnostic guidelines for primary ciliary dyskinesia: similarities and differences in approach to diagnosis. Eur Respir J 2019; 54: 1901066 [https://doi.org/10.1183/13993003.01066-2019].

\begin{abstract}
Introduction
Primary ciliary dyskinesia (PCD) is a genetically and clinically heterogeneous disease, usually inherited in an autosomal recessive pattern. Patients with PCD develop recurrent and chronic infections of upper and lower airways, invariably leading to bronchiectasis and impaired lung function. Conductive hearing impairment is common and half of people with PCD have situs abnormalities, e.g. situs inversus or situs ambiguus, which can be associated with congenital heart disease. Many, but not all men are infertile due to immotile sperm, and some women are sub-fertile because of immotile cilia in the Fallopian tubes [1].

Early diagnosis facilitates treatment with an aim to delay the onset of bronchiectasis, reduce loss of lung function, optimise hearing and manage symptoms of rhinosinusitis [2]. However, diagnosis is often missed or delayed, and until recently, there were no standards for confirming or refuting a diagnosis [3-5]. Therefore, the European Respiratory Society (ERS) commissioned a task force to provide guidelines for the diagnosis of PCD in 2017 [6], while the American Thoracic Society (ATS) published a practice guideline in 2018 [7]. Both guidelines were evidence-based, yet appear to present different recommendations. To reduce confusion, some of the authors from the two guidelines have met to discuss the similarities and differences and highlight steps required to improve agreement on diagnostic criteria. Here we aim to explain the reasons for different approaches in North America and Europe, and to clarify aspects that we know are causing confusion.
\end{abstract}

Methodology and application of the grade system in PCD guideline development Both ERS and ATS developed guidelines based on pre-defined questions relevant for clinical care, a systematic review of the literature, and assessment of the evidence using the GRADE approach to decide 
a)

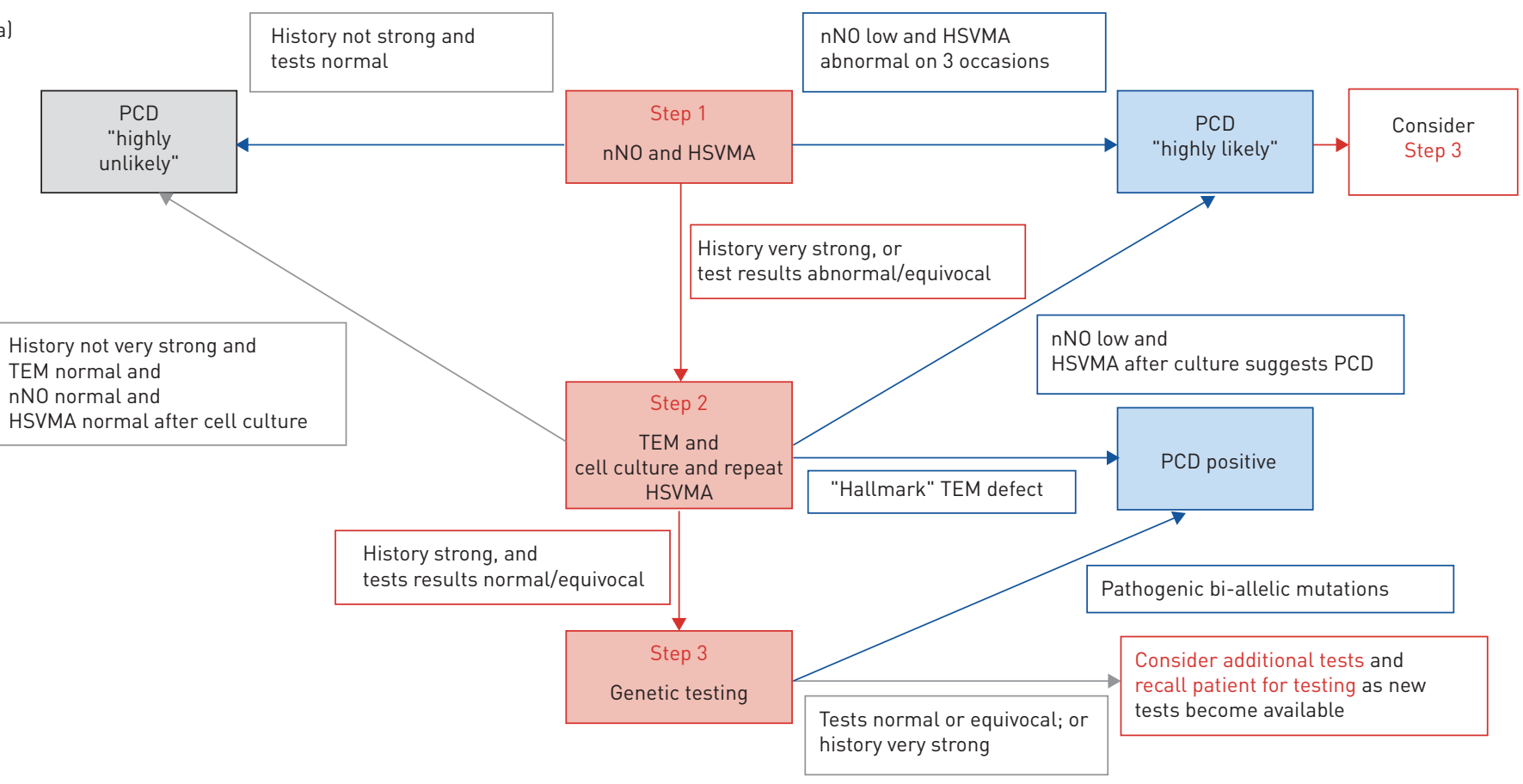

b)

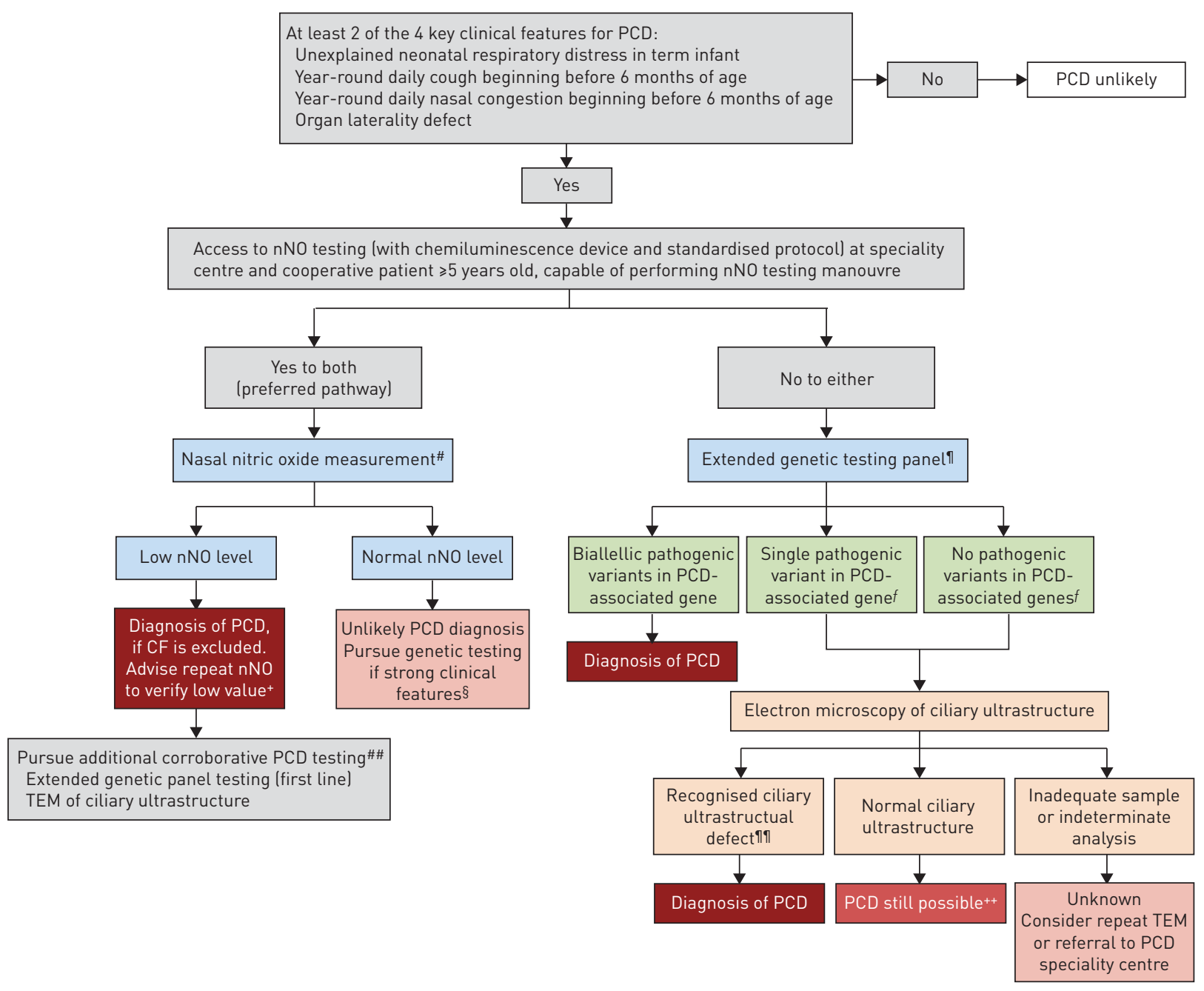


FIGURE 1 a) Adapted from the European Respiratory Society primary ciliary dyskinesia (PCD) diagnostic algorithm [6]. Patients do not necessarily need to go through all steps, and the order of investigations may vary depending on local resources [6]. The clinical importance of the algorithm is the summary of how the combination of tests contributes to the diagnostic outcome: positive, highly likely and highly unlikely. b) Adapted from the American Thoracic Society PCD diagnostic algorithm [7]. \#: cystic fibrosis (CF) should be ruled out before performing nasal nitric oxide (nNO) measurement, as roughly one-third of CF patients can have nNO values below PCD diagnostic cut-offs. nNO measurements should only be performed with chemiluminescence analysers using standardised protocols at centres with specific expertise in nNO measurements. Some nNO analysers have not received approval from federal agencies worldwide (US Food and Drug Administration and Health Canada), which may have implications for clinical implementation. ": genetic panel testing for mutations in $>12$ disease-associated PCD genes, including deletion/duplication analysis. ${ }^{+}$: as nNO levels can be significantly decreased by viral respiratory tract infections, a repeat nNO measurement, at least 2 weeks after the initial low value (expert opinion), is recommended to ensure the initial low value is not secondary to a viral process. A normal nNO value upon repeat testing is not suggestive of PCD, as nNO values remain consistently low in PCD. ${ }^{\S}$ : most forms of PCD resulting in normal $\mathrm{nNO}$ levels have normal or non-diagnostic electron microscopy studies. Thus, genetic testing is recommended in these cases. ${ }^{f}$ : or presence of variants of unknown significance. \#\#: additional corroborative testing may provide information on clinical prognosis, further disease understanding, and potential future therapeutic considerations. "ก: known disease-associated transmission electron microscopy (TEM) ultrastructural defects include outer dynein arm (ODA) defects, ODA plus inner dynein arm (IDA) defects, IDA defect with microtubular disorganisation, and absent central pair, identified using established criteria $[1,6,13]$. Of note, the presence of IDA defects alone is rarely diagnostic for PCD. ${ }^{++}$: up to $30 \%$ of PCD cases can have normal ciliary ultrastructure on electron microscopy. Consider referral to PCD specialty centre if there is a strong clinical phenotype but all electron microscopy and genetic testing are negative. HSVMA: high-speed video microscopy analysis.

the strength of recommendations (Grading of Recommendations, Assessment, Development and Evaluation) [8-11]. Both committees comprised international multidisciplinary PCD experts, expert methodologists and patient representatives.

For the ERS guideline, the predefined "PICO" question for each diagnostic test was: with Patients suspected of having PCD, Investigated by nasal nitric oxide (nNO), transmission electron microscopy (TEM), high-speed video microscopy analysis (HSVMA), genetics or immunofluorescence labelling, when Comparing patients with a final positive or negative outcome, what was the diagnostic accuracy (Outcome) of the test? To determine the value of each diagnostic test, the task force required studies that mimic the clinical situation. They therefore only included studies with patients referred for PCD testing, in whom the diagnosis was initially uncertain. Case-control studies that compared previously diagnosed typical PCD patients with healthy controls or disease controls were not considered because the situation does not occur in clinical practice.

The ATS objective determined the accuracy of four ancillary diagnostic tests (an extended genetic panel test including $>12$ PCD genes, nNO, HSVMA and ciliary beat frequency) for diagnosing PCD in patients with a high pre-test probability of having PCD. Studies that recruited patients with at least two of four clinical features (early onset chronic wet cough, early onset chronic nasal congestion, unexplained neonatal respiratory distress, or organ laterality defects) met criteria for high pre-test likelihood of PCD. Committee members agreed on a reference standard diagnosis for PCD: either a classic cilia ultrastructural defect detected on TEM and/or two pathogenic variants in one disease causing PCD gene. Four PICO questions were developed which compared the four ancillary PCD tests as replacements of the reference standard diagnosis. For the nNO PICO question, the population was limited to patients $\geqslant 5$ years of age with cystic fibrosis ruled out.

Only a small number of published studies met the strict GRADE criteria for inclusion in the guidelines. For example, the ERS systematic search identified 98 manuscripts about $\mathrm{nNO}$, but only four met the inclusion criteria for study design. None of the 462 studies on genetics, nor 276 studies reporting immunofluorescence met the criteria. There is therefore an urgent need for well-designed studies in a diagnostic setting to better inform future revisions of the guidelines.

\section{Summary of the ERS and ATS guidelines}

The ERS and ATS guidelines are summarised in figure 1 and comparisons between the two guidelines detailed in table 1.

The decision of whether a patient has a definitive diagnosis of PCD is not always straightforward, and both the ERS and ATS task forces concluded that there is no stand-alone test or combination of tests that can exclude PCD with $100 \%$ certainty in a patient with strong clinical phenotype. The ERS guidelines determine three diagnostic categories: "PCD confirmed", "PCD highly likely" and "PCD highly unlikely". If the diagnosis is "highly likely" but not conclusive, patients should be told that the diagnosis is likely, but given the limitations of diagnostic tests, patients should have other causes for their symptoms investigated whilst receiving treatment for PCD until a definite cause can be established. As new diagnostic tests become available, further investigations should be offered. ATS guideline diagnoses are more binary with "PCD positive" and "PCD negative". 
TABLE 1 Comparison of recommendations for the diagnosis of primary ciliary dyskinesia (PCD) by the European Respiratory Society (ERS) task force and American Thoracic Society (ATS) guideline committee

ERS task force (2017) [6]

Final guideline criteria endorsed for definitive PCD diagnosis

Patients included/referred for testing

\section{Evidence based recommendations for the role of diagnostic tests in patients with high pre-test probability of PCD\# (GRADE strength of recommendations)}

Nasal nitric oxide (nNO)

Ciliary ultrastructure analysis by transmission electron microscopy (TEM)

Genetic testing

High speed video microscopy (HSVMA)

Ciliary beat frequency (CBF)

Immunofluorescence (MODERATE).
Biallelic pathogenic variants in PCD-associated gene or TEM hallmark ciliary ultrastructural defect.

A combination of typical symptoms, possibly supported by symptom-based predictive tools such as PICADAR [12]. PICADAR score of 4 has sensitivity 0.97 , specificity 0.48 ; score of 5 has a sensitivity of 0.90 and specificity of 0.75 [12].

ATS guideline committee (2018) [7]

Biallelic pathogenic variants in PCD-associated gene or TEM hallmark ciliary ultrastructural defect or low nNO level ${ }^{+}$

Two of four clinical features: early onset chronic wet cough, early onset chronic nasal congestion, unexplained neonatal respiratory distress or an organ laterality defect (for two key symptoms: sensitivity 0.80 , specificity $0.72 \%$; for four key symptoms: sensitivity 0.21 specificity 0.99) [15].

$\mathrm{nNO}^{\text {Il }}$ should be used as part of the diagnostic work-up of patients $>6$ years

TEM should be used as part of the diagnostic work-up (STRONG). Further diagnostic testing should be performed in patients with normal TEM if clinical history is strong (STRONG). A hallmark defect confirms diagnosis (STRONG). No studies met inclusion criteria for analysis.

HSVMA should be used as part of the diagnostic work-up (WEAK). HSVMA should be repeated after ALI culture (STRONG).

CBF alone should not be used as part of the diagnostic work-up (STRONG).

No studies met inclusion criteria for analysis.
Low $\mathrm{nNO}^{+}$should be used to replace TEM and/or PCD gene testing in cooperative patients $\geqslant 5$ years, when CF ruled out (CONDITIONAL).

TEM not evaluated as it was included in the diagnostic reference standard.

An extended gene panel (>12 genes) should be used to replace electron microscopy and/or standard gene panels ( $\leqslant 12$ genes) (CONDITIONAL).

HSVMA should not be used to replace electron microscopy and/or PCD gene testing (CONDITIONAL).

CBF alone should not be used to replace electron microscopy and/or PCD gene testing (CONDITIONAL).

Not evaluated.

\footnotetext{
\#: ERS task force evaluated specific clinical criteria to develop evidence-based recommendations for which patients should be referred for diagnostic testing, while ATS guideline committee specified criteria a priori. " : preferably using a chemiluminescence analyser with a velum closure technique. In children <6years, tidal breathing should be used as part of the diagnostic work-up (weak recommendation) ${ }^{+}:<77 \mathrm{~nL} \cdot \mathrm{min}^{-1}$ measured on two separate occasions with a chemiluminescence device using a standardised protocol at a PCD specialty centre. Corroborative testing with TEM and/or genetic testing should be pursued to further define PCD phenotype and genotype, which may assist in clinical prognosis and family planning. CF: cystic fibrosis; ALI: air-liquid interface.
}

\section{Explanation of some differences between ATS and ERS guidelines}

In box 1 we propose reasons why the use of the same evidence has led to different recommendations. First, the ATS guidelines had a strict clinical criteria compared to the ERS, thus altering pre-test probability of a false-positive or false-negative result. The evidence has also been evaluated on a background of different geographical challenges and healthcare systems, and therefore accessibility, availability and feasibility of testing has impacted the recommendations. For example, in Europe, where HSVMA and TEM equipment is established and expertise is concentrated in national referral centres, these tests are feasible, whereas in North America, access to these tests is more limited as PCD infrastructure is less centralised and spread across a vast and geographically dispersed medical system, where feasibility is challenged through lack of experience across institutions. Conversely, in North America, clinical genotyping is already recognised through insurance systems as a regulated and reliable test. Interestingly, both guideline committees included patient representatives and their different 


\section{Box 1}

\section{Possible explanations for differences in guidelines}

- Different PICO question formats

- Different reference standard for comparison to index test

- Different outcomes: highly likely, confirmed, highly unlikely PCD diagnosis versus binary (positive or negative PCD diagnosis)

- Different included studies (additional year of published evidence for ATS guidelines)

- Different grading classification of evidence: strong, moderate, weak versus binary (strong or conditional)

- Different healthcare and insurance systems (e.g. funding for HSVMA versus genetic testing)

- Different geography for access to testing

- Different government regulations for approving new clinical diagnostic testing (e.g. medical device approval for nNO testing, CLIA-certified labs for genetic testingl

Suggested research required to develop an internationally agreed diagnostic pathway for PCD

- International standardisation of operational procedures and quality controls for existing PCD diagnostic tests

- Demonstration of high feasibility for PCD diagnostic tests across academic and clinical centres

- Development of high-quality evidence (i.e. prospective cohort studies) for determining sensitivity and specificity of all diagnostic tests

- Standardised reporting of diagnostic test studies (i.e. using STROBE checklist, $\mathrm{nNO}$ values in $\mathrm{nL} \cdot \mathrm{min}^{-1}$ instead of concentration in ppb, disease-causing defects in TEM, disease-causing beat pattern classifications in HSVMAl

- Understanding genotype-phenotype relationships

- Further discovery of the genetic causes of PCD, including identifying and shared data-banking of pathogenic versus benign variants in each known PCD gene

- Development of new diagnostic testing modalities (e.g. immunofluorescence testing)

PCD: primary ciliary dyskinesia; ATS: American Thoracic Society; HSVMA: high-speed video microscopy analysis; nNO: nasal nitric oxide; CLIA: Clinical Laboratory Improvement Amendments; TEM: transmission electron microscopy.

experiences may also have influenced the committee. Possibly, the biggest differences are in the definition of the reference standard and the years that these guidelines were developed. The ATS had an additional year of peer reviewed publications which included a key cohort publication for genetic testing. Also, the ATS used updated GRADE guidelines that have a binary classification of recommendations (strong or conditional), and they were all conditional due to the lack of evidence that a diagnosis of PCD changes long-term health outcomes.

\section{Discussion}

There are strengths and weaknesses of all the diagnostic tests for PCD. Both guidelines agree that a diagnosis of PCD can be confirmed with hallmark cilia ultrastructural defects or a positive genetic test. However, neither test alone has good sensitivity. It is estimated that non-diagnostic ultrastructure by TEM accounts for up to $30 \%$ PCD cases, and that causative genetic mutations are not found in roughly the same number of patients [13-15].

Both guidelines agree there is a place for nNO testing in PCD diagnosis as it is quick and simple to perform and has good sensitivity and specificity when conducted according to standardised protocols [16]. However, it has been shown to be normal in an increasing number of cases confirmed by genotyping (e.g. CCDC103, RSPH1) [17, 18]. The ATS guideline states that PCD diagnosis can be confirmed with two low nNO values, whereas the ERS guideline states that low nNO only in combination with abnormal HSVMA makes the diagnosis highly likely (but not confirmed). The discriminatory value of the NO test is age-dependent and, therefore, is only recommended in cooperative patients $\geqslant 5$ years old. Diagnostic cut-off values for nNO remain poorly defined in younger children, who are unable to perform velum closure manoeuvres adequately and do not have fully formed paranasal sinuses. This group of children $<5$ years old are ultimately the target group for PCD testing since earlier diagnosis has been linked to improved disease outcome [19].

The guidelines differ vastly in their evaluation of ciliary function by HSVMA; it is central to the ERS and not recommended by the ATS guideline, perhaps reflecting existing clinical practices and differing PICO question formats; the ATS guidelines examined HSVMA as a stand-alone replacement of the reference standard tests while ERS guidelines evaluated HSVMA as a diagnostic test to be used in conjunction with other PCD testing modalities. Both guidelines suggest a shift from traditional measurements of ciliary beat frequency with universal agreement that this is not adequate. Rather, use of HSVMA to analyse effective 
mucociliary clearance and ciliary beat pattern has become increasingly used in European centres. Since the publications of the guidelines, the authors have worked towards providing better evidence for this test, yet HSVMA testing procedures and interpretations remain non-standardised across centres [20].

It is clear from the evidence presented in both guidelines that relying on just one or two tests will result in lower diagnostic accuracy in a minority of cases. With each additional test included in diagnostic algorithms, diagnostic accuracy is improved. With the tools currently available, the tolerance of lower diagnostic accuracy must be balanced with access to testing, feasibility of testing, cost of testing and, consequently, access to treatment. Use of $\mathrm{nNO}$ and a strict set of clinical characteristics, as suggested in the ATS guideline, may miss cases with normal NO or atypical symptoms, though the ATS diagnostic algorithm does suggest further PCD testing with strong clinical phenotypes, even when nNO levels are normal. The ERS guideline on the other hand never excludes a diagnosis and deems patients as PCD highly unlikely, leaving a more complex situation for counselling patients.

Finally, both guidelines concur with PCD diagnostic testing having the highest diagnostic accuracy when performed in centres with specific expertise in PCD. While genetic testing can be feasibly performed by any institution, the pre-test probability of a positive genetic result will be higher if other diagnostic tests, like nNO, are already positive for PCD. Variants of unknown significance are common in the multiple PCD-causing genes. Therefore, to avoid false positive diagnoses, expert interpretation of variants and confirmation of pathogenicity with other tests (HSVMA, TEM) are required to ensure the genotype is compatible with the abnormalities in ciliary function and ultrastructure. Ciliary biopsy samples for both TEM and HSVMA testing are difficult to correctly collect, process and interpret outside of highly specialised PCD centres. Thus, referral to PCD specialty centres, like the national referral centres across Europe and the PCD Foundation Clinical and Research Center Network in North America, will often be necessary for a definitive PCD diagnosis.

\section{Suggested research required to develop an internationally agreed diagnostic pathway for PCD}

Although there are many points of agreement, the disparities between the two guideline approaches are confusing for the end user, and in a rare disease such as PCD, an internationally agreed upon diagnostic testing guideline would be preferable. There are two purposes for the requirements of a clear PCD diagnostic definition: 1) to inform patients of their diagnosis and (where applicable) for insurance purposes to access treatment, and 2) for recruitment into research and drug development trials. Evidence for PCD treatment is severely lacking and usually borrowed from studies in cystic fibrosis or bronchiectasis. Whilst it is reasonable to have a relatively low threshold for starting clinical management for PCD based on low nNO, it must be recognised that there will be false-positive and false-negative cases, and that further confirmatory testing should be explored with genetics and TEM, as recommended in both guidelines. It is paramount for clinical studies that the correct patients are recruited, and only patients with definitive PCD diagnoses are included.

Box 1 highlights some suggestions for steps required to further develop international agreement. Significant research and development is required to identify new testing modalities and improve those already in use. New modalities currently being investigated include radiolabelled mucociliary clearance testing and use of immunofluorescence [21]. Funding for PCD genetic testing across European national health care programmes should also be strongly advocated.

Finally, PCD is a heterogenous group of genetic conditions and it may be that one test or one algorithm will never fit all cases. Large gene discovery programmes and cohort studies such as the European iPCD study and the Genetic Disorders of Mucociliary Clearance Consortium (GDMCC) will in future allow improved understanding of molecular causes of disease and consequently PCD diagnosis [22].

Conflict of interest: A. Shoemark has nothing to disclose. S. Dell is site PI for the CLEAN PCD study, Parion Sciences. A. Shapiro has nothing to disclose. J.S. Lucas reports personal fees for attending meetings and research, and has been a member of a PCD advisory board for Aerocrine/Circassia, outside the submitted work.

\section{References}

1 Goutaki M, Meier AB, Halbeisen FS, et al. Clinical manifestations in primary ciliary dyskinesia: systematic review and meta-analysis. Eur Respir J 2016; 48: 1081-1095.

2 Lucas JS, Alanin MC, Collins S, et al. Clinical care of children with primary ciliary dyskinesia. Expert Rev Respir Med 2017; 11: 779-790.

3 Behan L, Dunn Galvin A, Rubbo B, et al. Diagnosing primary ciliary dyskinesia: an international patient perspective. Eur Respir J 2016; 48: 1096-1107.

4 Kuehni CE, Frischer T, Strippoli MP, et al. Factors influencing age at diagnosis of primary ciliary dyskinesia in European children. Eur Respir J 2010; 36: 1248-1258. 
5 Kuehni CE, Lucas JS. Diagnosis of primary ciliary dyskinesia: summary of the ERS Task Force report. Breathe (Sheff) 2017; 13: 166-178.

6 Lucas JS, Barbato A, Collins SA, et al. European Respiratory Society guidelines for the diagnosis of primary ciliary dyskinesia. Eur Respir J 2017; 49: 1601090.

7 Shapiro AJ, Davis SD, Polineni D, et al. Diagnosis of Primary Ciliary Dyskinesia. An Official American Thoracic Society Clinical Practice Guideline. Am J Respir Crit Care Med 2018; 197: e24-e39.

8 Schunemann HJ, Oxman AD, Brozek J, et al. Grading quality of evidence and strength of recommendations for diagnostic tests and strategies. BMJ 2008; 336: 1106-1110.

9 Balshem $\mathrm{H}$, Helfand M, Schunemann HJ, et al. GRADE guidelines: 3. Rating the quality of evidence. $J$ Clin Epidemiol 2011; 64: 401-406.

10 Guyatt GH, Oxman AD, Kunz R, et al. GRADE guidelines: 2. Framing the question and deciding on important outcomes. J Clin Epidemiol 2011; 64: 395-400.

11 Andrews JC, Schunemann HJ, Oxman AD, et al. GRADE guidelines: 15. Going from evidence to recommendation-determinants of a recommendation's direction and strength. J Clin Epidemiol 2013; 66: 726-735.

12 Behan L, Dimitrov BD, Kuehni CE, et al. PICADAR: a diagnostic predictive tool for primary ciliary dyskinesia. Eur Respir J 2016; 47: 1103-1112.

13 Boon M, Smits A, Cuppens H, et al. Primary ciliary dyskinesia: critical evaluation of clinical symptoms and diagnosis in patients with normal and abnormal ultrastructure. Orphanet J Rare Dis 2014; 9: 11.

14 Paff T, Kooi IE, Moutaouakil Y, et al. Diagnostic yield of a targeted gene panel in primary ciliary dyskinesia patients. Hum Mutat 2018; 39: 653-665.

15 Leigh MW, Ferkol TW, Davis SD, et al. Clinical features and associated likelihood of primary ciliary dyskinesia in children and adolescents. Ann Am Thorac Soc 2016; 13: 1305-1313.

16 ATS/ERS recommendations for standardized procedures for the online and offline measurement of exhaled lower respiratory nitric oxide and nasal nitric oxide, 2005. Am J Respir Crit Care Med 2005; 171: 912-930.

17 Knowles MR, Ostrowski LE, Leigh MW, et al. Mutations in RSPH1 cause primary ciliary dyskinesia with a unique clinical and ciliary phenotype. Am J Respir Crit Care Med 2014; 189: 707-717.

18 Shoemark A, Moya E, Hirst RA, et al. High prevalence of CCDC103 p.His154Pro mutation causing primary ciliary dyskinesia disrupts protein oligomerisation and is associated with normal diagnostic investigations. Thorax 2018; 73: 157-166.

19 Marthin JK, Philipsen MC, Rosthoj S, et al. Infant nasal nitric oxide over time: natural evolution and impact of respiratory tract infection. Eur Respir J 2018; 51: 1702503.

20 Rubbo B, Shoemark A, Jackson CL, et al. Accuracy of high-speed video analysis to diagnose primary ciliary dyskinesia. Chest 2019; 155: 1008-1017.

21 Shoemark A, Frost E, Dixon M, et al. Accuracy of immunofluorescence in the diagnosis of primary ciliary dyskinesia. Am J Respir Crit Care Med 2017; 196: 94-101.

22 Goutaki M, Maurer E, Halbeisen FS, et al. The international primary ciliary dyskinesia cohort (iPCD Cohort): methods and first results. Eur Respir J 2017; 49: 1601181. 